Research Paper

\title{
Prognostic role of NF-YA splicing isoforms and Lamin A status in low grade endometrial cancer
}

\author{
Lucia Cicchillitti, ${ }^{1, *}$, Giacomo Corrado ${ }^{2, *}$, Mariantonia Carosi $^{3}$, Malgorzata Ewa \\ Dabrowska ${ }^{3}$, Rossella Loria ${ }^{1}$, Rita Falcioni ${ }^{1}$, Giuseppe Cutillo ${ }^{2}$, Giulia Piaggio ${ }^{1, * *}$, \\ Enrico Vizza ${ }^{2, * *}$ \\ ${ }^{1}$ Department of Research, Advanced Diagnostics and Technological Innovation, Area of Translational Research, Regina Elena \\ National Cancer Institute, Rome, Italy \\ ${ }^{2}$ Department of Experimental Clinical Oncology, Gynecologic Oncology Unit, Regina Elena National Cancer Institute, Rome, \\ Italy \\ ${ }^{3}$ Department of Research, Advanced Diagnostics and Technological Innovation, Anatomy Pathology Unit, Regina Elena \\ National Cancer Institute, Rome, Italy \\ *These authors have contributed equally to this work \\ ** co-last authors \\ Correspondence to: Giacomo Corrado, email: giacomo.corrado@alice.it \\ Lucia Cicchillitti, email: luciacicchi@gmail.com \\ Keywords: endometrial cancer, lamin A, NF-Y, estrogen receptor, miR-200 family \\ Received: July 02, $2016 \quad$ Accepted: November 14, $2016 \quad$ Published: December 10, 2016
}

\section{ABSTRACT}

Although most cases of low grade (G1) endometrial cancer (EC) do not behave aggressively, in rare instances, can progress in a highly aggressive manner. In this study we analyzed formalin-fixed, paraffin-embedded (FFPE) EC tissues to find novel clinical and biological features to help diagnosis and treatment of G1 ECs $s$ in order to better stratify patient risk of recurrence. A retrospective cohort of FFPE specimens from patients with EC $(n=87)$ and benign tissue specimens (NE) from patients who underwent a hysterectomy to treat other benign disease $(n=13)$ were collected. Total RNA and proteins were extracted and analyzed, respectively, by quantitative PCR and western blotting. NF-YAs is expressed and lamin A is down-modulated in all high grade (G2 and G3) ECs. In G1 ECs, NF-YAs expression is heterogeneous being expressed only in a subset of these tumours. Interestingly, the G1 ECs that express NF-YAs display low levels of lamin A similar to those present in G2 and G3 ECs. Of note, this pattern of NF-YAs and lamin A expression correlates with tumor aggressiveness assessed by comparative analysis with estrogen receptor (ER) status and epithelial-mesenchymal transition (EMT) markers thus suggesting its potential role as biomarker of tumour aggressiveness in G1 EC. In all grade ECs, lamin A is strongly downmodulated, being its expression inversely correlated with tumor aggressiveness and its loss of expression. We identified NF-YAs and lamin A expression levels as novel potential biomarkers useful to identify G1 ECs patients with risk of recurrence.

\section{INTRODUCTION}

Endometrial cancer (EC) is the most common genital tract malignancy and occurs in reproductive and postmenopausal women. Most EC cases are sporadic, with only $10 \%$ considered familiar [1]. In general, patients with EC have a good prognosis since early diagnosis is frequent and the disease has usually not spread beyond the uterus. However, the prognosis for recurrent or metastatic
EC remains poor and in order to improve therapy it is important to understand the processes which inhibit and stimulate cancer progression. EC is classified as type I or type II based on histologic properties. Type I, also called the endometrioid type (EEC) because of its histologic similarity to the endometrium, accounts approximately $70-80 \%$ of sporadic EC. Most type I tumors occur in the setting of unopposed estrogen stimulation, leading to endometrial hyperplasia usually classified as grade 1 , 
2 or 3 (G1, G2 or G3) depending on their histological similarity to endometrium[1]. Unlike type I tumors, type II lesions are usually G3 and are not related to estrogen exposure or endometrial hyperplasia and include high risk malignancies, as serous papillary and clear cell carcinoma, generally [1]. Identification of patients with poor prognosis among the presumed low risk, G1 endometrioid cases represents a particular therapeutic challenge. Several prognostic factors, such as histological type, histological grade, surgical stage, pelvic lymph node involvement and myometrial invasion have been established $[2,3$, 4], and [5]. Meanwhile, some biological molecules have been identified as prognostic markers in EC, such as KRAS, PTEN, EGFR, FGFR, P53, HER2, and estrogen receptors (ERs) [6], but more efforts are needed to identify novel biomarkers with a potential for a more systematic integration in clinical practice for individualized therapy in EC.

Nuclear transcription factor (NF-Y) is transcription factor that activates genes involved in growth promotion including cell cycle regulatory genes. Numerous findings highlight that NF-Y is involved in cancer [7], [8], [9] and [10]. NF-Y is composed of three different subunits: YA, YB and YC. Subunit NF-YA has two different isoforms, NF-YAl (long) and NF-YAs (short), resulting from alternative splicing. Previous studies demonstrated that NF-YAs is down-regulated, whereas NF-YAl is upregulated, during differentiation of hESCs, mouse ES cells, and hematopoietic stem cells [11], [12] and [13]. Although mutations in NF-Y subunits have never been specifically identified in tumours, systematic examination of protein expression profiles indicates that NF-Y targets are upregulated in different types of cancer. Recently, informatics analysis and microarray expression profile studies conducted in various gynecological cancers, revealed that also in these tumors, several NF-Y target genes are upregulated [14].

Expression of ERs has been correlated with EC stage, histologic grade and survival [15]. Loss of ERs has been significantly associated with aggressive phenotype and poor survival in EC patients [16]. In particular, early stage, well differentiated ECs usually retain ERs expression, whereas advanced stage, poorly differentiated tumours often lack one or both receptors. In the human uterus, ER- $\alpha$ is the predominant subtype [17], and ER- $\beta$ is supposed to play an $i$ role by modulating ER- $\alpha$ function [18] and [19]. Recently, a link between hormone receptor status and epithelialmesenchymal transition (EMT) has been recently proposed [20]. EMT enables epithelial tumor cells to acquire a like mesenchymal potential with increase motility and ability to extravasate and circulate. In EC, alteration of EMT markers, including several miRNAs, including miR200 family, have been identified in metastatic disease and associated with reduced survival [20].

Nuclear lamins are type $\mathrm{V}$ intermediate filament proteins that are critically for the structural properties of the nucleus and are involved in cell migration and differentiation [21], and [22]. A-type lamins, whose most represented isoforms are lamin A and $\mathrm{C}$, are alternatively spliced products of the same gene. Loss of lamin A expression has been reported for several types of cancers [22], [23], and [24], recently also for also for EC [25].

In the present study, we assessed NF-YA isoforms and lamin A expression in several EC samples and identified them as novel potential prognostic EC biomarkers. We report for the first time that a specific NF-YA splicing isoform, NF-YAs, is associated with EC development. In our cohort of G1 EECs, NF-YAs was expressed in about $55 \%$ of samples, whereas it was detectable in all higher grade and non endometrioid (NEM) ECs. Also, our data indicate that an association between NF-YAs isoform and lower ER- $\alpha$ mRNA (ESR1) expression occurs. Interestingly, clustering of NF-YA isoforms in G1 EEC indicated an inverse association between NF-YAs and lamin A expression and a direct correlation with an increase of miR-200 levels.

Our findings suggest NF-YAs and lamin A as novel biomarkers with a potential for a more systematic integration in clinical practise for individualised therapy in EC, in particular in low grade malignancy.

\section{RESULTS}

\section{NF-YA splicing isoforms are differentially expressed in EC tissues}

We analysed the expression of NF-YA and NFYB subunits in several EC FFPE specimens, whose clinic-pathological features are described in Table 1, and compared the protein expression level of the two subunits in EC and NE FFPE specimens (Figure 1A).

We did not detect any significant modulations of NF-YA and NF-YB protein levels in ECs compared to NE tissues but, very interestingly, we observed a different and specific electrophoretic profile of NFYA splicing isoforms. We found that in NE tissues the long isoform of NF-YA (NF-YAl) was the main form expressed, whereas the short isoform (NF-YAs) was mostly undetectable. Interestingly, NF-YAs was clearly detectable in EC tissues (Figure 1A), suggesting its specific association with a tumour phenotype. NF-YAs was expressed in all higher grades, G2 and G3 EEC, and NEM tissues, whereas it was detected only in $55 \%$ of our G1 samples (G1). This result suggests that NF-YAs could represent a marker of EC and, also, an indicator of tumour aggressiveness. Assessment of the ratio of mRNA expression of NF-YAs and NF-YAl (NF-YAs/NF-YAl) by qRT-PCR using primers designed to specifically amplify only NF-YAl or NF-YAs mRNA was very similar in NE and G1 EEC tissues (Figure 1B), thus indicating that protein levels of NF-YA isoforms very likely depend on post-transcriptional mechanisms. 
Table 1: Clinicopathological features of 87 ECs

\begin{tabular}{lcccc}
\hline $\begin{array}{l}\text { Clinico-pathological } \\
\text { features }\end{array}$ & Total EECs & G1 EECs & G2-G3 EECs & NEM \\
\hline $\begin{array}{l}\text { No of cases } \\
\text { Median Age } \\
\text { (years) }\end{array}$ & 78 & 29 & 49 & 9 \\
BMI > 30 & 63 (range $42-88)$ & 58 (range 42-76) & 66 (range 43-88) & 65 (range 50-74) \\
MI > 50\% & $14(17.9 \%)$ & $8(27.5 \%)$ & $6(12.2 \%)$ & $7(77.7 \%)$ \\
Lymph node metastases & $33(42.3 \%)$ & $5(17.2 \%)$ & $28(57.1 \%)$ & $7(77.7 \%)$ \\
FIGO Stage & $4(5.1 \%)$ & $0(0.0 \%)$ & $4(8.1 \%)$ & $2(22.2 \%)$ \\
I and II & $40(51.2 \%)$ & $29(100 \%)$ & $21(42.8 \%)$ & $6(66.6 \%)$ \\
III and IV & $28(35.9 \%)$ & $0(0.0 \%)$ & $28(57.1 \%)$ & $3(33.3 \%)$ \\
Site of recurrence & & & & \\
Local & $17(21.8 \%)$ & $0(0.0 \%)$ & $17(34.7 \%)$ & $0(0.0 \%)$ \\
Distant & $10(12.8 \%)$ & $2(6.9 \%)$ & $8(16.3 \%)$ & $0(0.0 \%)$ \\
Multifocal & $14(17.9 \%)$ & $0(0.0 \%)$ & $14(28.6 \%)$ & $3(33.3 \%)$ \\
RT & $29(37.1 \%)$ & $3(10.3 \%)$ & $26(53.0 \%)$ & $4(44.4 \%)$ \\
CHT & $24(30,7 \%)$ & $0(0.0 \%)$ & $15(30.6 \%)$ & $9(100 \%)$ \\
\hline
\end{tabular}

$\mathrm{RT}=$ adjuvant radioteraphy; $\mathrm{CHT}=$ adjuvant chemoteraphy; $\mathrm{BMI}=$ body mass index;

$\mathrm{MI}=$ myometrial infiltration.

A

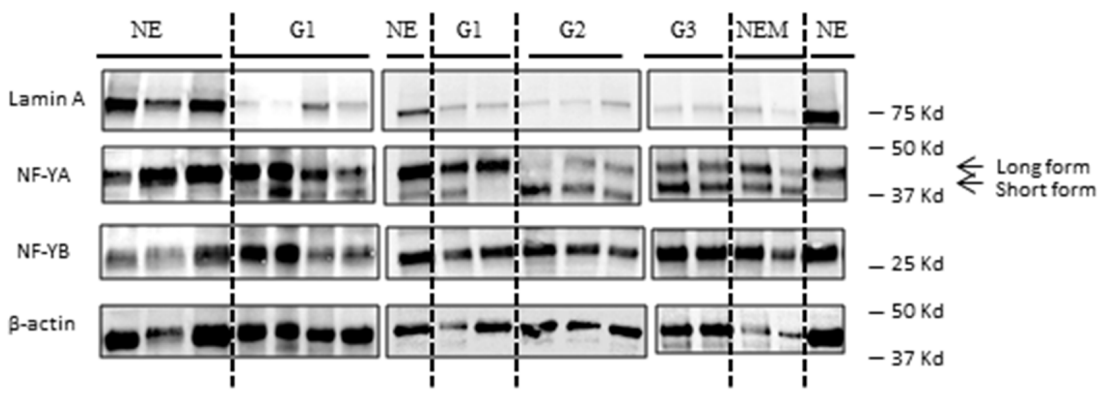

B

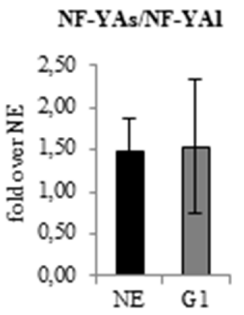

Figure 1: Analysis of NF-YA isoforms and lamin A protein expression in benign and EC tissues. A. Representative immunoblottings of proteins extracted from benign (NE), low grade G1 (G1) and high grade endomentrial endometrioid cancer (G2-G3) and non endometrioid (NEM) EC FFPE tissues with anti NF-YA, anti-NF-YB, anti-Lamin A antibodies. The dashed lines separate the different groups. Anti- $\beta$ actin was used as loading control. B. Average expression of the ratio NF-YAs to NF-YAl mRNA expression examined by qRT$\mathrm{PCR} \pm \mathrm{SD}$ in G1 EEC tissues. mRNA expression was normalized for $18 \mathrm{~S}$ rRNA levels. The error bars indicate the standard error. 


\section{NF-YAs detection in G1 EEC tissues correlates with decreased ERs mRNA expression}

We first analysed ERs status to evaluate its prognostic values in our patient cohort (Table 2). In G1 EEC, 27.5\% and $31 \%$ of tissues were found to express low ER- $\alpha$ (ESR1) and ER- $\beta$ (ESR2) mRNA levels, respectively. A large downmodulation of ESR1 and ESR2 mRNA, in $61.2 \%$ and $77.5 \%$ of high grade EC and $77.7 \%$ and $88.8 \%$ NEM samples respectively, was observed (Table 2), thus indicating that reduction of ERs level is related with advanced stage of EC. It is worth noting that ESR2 mRNA expression levels were very variable in our cohort of specimens. Based on these evidences, we focused on ESR1 status to correlate its expression level in EC subtypes. Results displayed a correlation of reduction of ESR1 expression with an aggressive clinicopathologic phenotype (Figure 2A). Then, we focused our attention on ESR-1 mRNA expression and the expression of NF-YA isoforms in G1 EECs, where a heterogeneous NF-YA expression of the two splicing isoforms was observed. We observed an association between the presence of NF-YAs and lower ESR1 mRNA levels (Figure 2A and Table 2), thus indicating a possible involvement of NF-YAs in EC aggressiveness. It is worth to note that ESR1 mRNA levels in NF-YAs positive tissues were very similar to that of higher grades (G2 and G3), whereas in NF-YAs negative (NF-YAs-) specimens were comparable to those of NE tissues (Figure 2 and Table 2). These results strongly support a correlation between ERs status and NF-YAs expression.

\section{Upregulation of miR-200 family inversely correlates with ZEBs expression in ECs and is related to NF-YAs expression in G1 EEC}

Lack of ER- $\alpha$ has been recently associated with EMT [26]. The miR-200 family has been extensively studied with respect its role in regulating genes of EMT. It is worth noting that upregulation of miR-200a and ERs loss have been associated with poor prognosis [29]. Analysis of the qRT-PCR data showed that all members of the miR-200 family analysed (miR-200a, miR-200b, miR-200c, and miR-141) were up-regulated in all stages of EC compared to NE tissues (Figure 3A), which confirms results reported in previous studies [27, 28 ] and [29]. In order to validate the activity of miR200 s in EC, we also analysed the expression level of ZEB1 and ZEB2 (Figure 3B), well established as direct targets of miR-200 family. In EC, levels of ZEB1 and ZEB2 were lower and further decreased in NEM, thus supporting the hypothesis of an augmented translational activity of miR-200 family members in more aggressive phenotype. Then, we focused our analysis in our subset of G1 samples. Interestingly, we observed a consistent increase of miR-200 family expression (Figure 3C) inversely related to ZEBs mRNA levels in NF-YAs positive compared with negative NF-YAs G1 EEC samples (Figure 3D), thus supporting the hypothesis of a correlation between NF-YAs expression and tumour aggressiveness. Finally, to better characterize the possible EMT involvement in G1 EEC, we assessed N-cadherin mRNA expression ( $\mathrm{CDH} 2)$, a mesenchymal marker, by qRT-PCR, and the ratio of E-cadherin to N-cadherin $(\mathrm{E} / \mathrm{N})$, an index of differentiated phenotype. Levels of $\mathrm{CDH} 2$ were augmented in all EC tissues and a further significant increase was observed in more aggressive phenotypes (Figure 2B). Moreover, as shown in Table 2 , we found that $31 \%, 52 \%$, and $44 \%$ of cases displayed low E/N index in G1, G2-G3 and NEM, respectively, thus indicating a trend towards EMT in more aggressive ECs. Analysis on our G1 tissues displayed the lack of any modulation of both $\mathrm{CDH} 2 \mathrm{mRNA}$ levels and $\mathrm{E} / \mathrm{N}$ ratio in NF-YAs positive compared with NF-YAs negative (Figure 2B and Table 2), suggesting that $\mathrm{CDH1}$ and $\mathrm{CDH} 2$ expression is not very likely associated with differential expression of NF-YA isoforms in G1 EEC.

\section{Decreased lamin A levels are associated with EC progression and aggressiveness and NF-YAs expression in G1 EEC}

Lamin A has been reported to be a direct target of miR-9 and that upregulation of miR-9 expression occurs in EC [30]. We assessed lamin A protein (Figure 1A) and mRNA expression (Figure 4A), and miR-9 levels (Figure 4B) in our cohort of EC tissues. Results displayed loss of lamin A expression in EC and that reduced lamin A mRNA levels were associated with overexpression of miR-9 (Figure 4B). Interestingly, data obtained by comparing lamin A mRNA levels in the two subgroups of G1 EEC tissues indicated that lamin $A$ reduction was significantly higher $(P \leq .01)$ in NF-YAs positive with respect to NFYAs negative samples (Figure 4A). Moreover, levels of lamin A in NF-YAs positive tissues were comparable to that of high grade EEC. These evidences further support the potential role of NF-YAs and lamin A as molecular indicator of tumour aggressiveness in early stage EC.

\section{DISCUSSION}

A recent study based on both informatics analysis and experimental evidence identified NF-Y as one of the key components of the transcription deregulation in gynecological cancer [14]. In agreement, here we identified a specific splicing isoform of the regulatory subunit of NF-Y, NF-YA, as a new potential indicator of aggressiveness in low grade G1 EEC. Indeed, although most cases of G1 EEC do not behave aggressively, in rare instances, even low-grade, well-differentiated endometrial adenocarcinomas can progress in a highly aggressive manner. We observed that NF-YA short isoform (NF-YAs) was undetectable in benign 
Table 2: EC histologic grade in relation to low levels of LMNA, ESR1, ESR2 expression, and E/N index

\begin{tabular}{|c|c|c|c|c|}
\hline $\begin{array}{l}\text { ERs, LMNA and E/N } \\
m R N A \text { expression in } \\
E C\end{array}$ & $\begin{array}{c}\text { Low ESR1 } \\
\text { n (\%) }\end{array}$ & $\begin{array}{c}\text { Low ESR2 } \\
\text { n (\%) }\end{array}$ & $\begin{array}{c}\text { Low LMNA } \\
\text { n }(\%)\end{array}$ & $\begin{array}{c}\text { Low E/N } \\
\text { n }(\%)\end{array}$ \\
\hline $\mathrm{NE}(\mathrm{n}=13)$ & $0(0 \%)$ & $6(46.1 \%)$ & $0(0 \%)$ & $2(15.3 \%)$ \\
\hline G1 EEC $(n=29)$ & $8(27.5 \%)$ & $9(31 \%)$ & $20(68.9 \%)$ & $9(31 \%)$ \\
\hline $\begin{array}{l}\text { G1 EEC NF-YAs- } \\
(n=13)\end{array}$ & $3(23 \%)$ & $3(23 \%)$ & $5(38.5 \%)$ & $4(30.7 \%)$ \\
\hline $\begin{array}{l}\text { G1 EEC NF-YAs }+ \\
(n=16)\end{array}$ & $5(31.2 \%)$ & $6(37.5 \%)$ & $15(93.7 \%)$ & $5(31.2 \%)$ \\
\hline G2-G3 EEC $(n=49)$ & $30(61.2 \%)$ & $38(77.5 \%)$ & $43(87.7 \%)$ & $25(52 \%)$ \\
\hline $\operatorname{NEM}(n=9)$ & $7(77.7 \%)$ & $9(88.8 \%)$ & $7(77.7 \%)$ & $4(44.4 \%)$ \\
\hline
\end{tabular}

EC histologic grade in relation to low levels of LMNA, ESR1, ESR2 expression, and E/N (CDH1/CDH2) index. LMNA, ESR1, ESR2, CDH1 and CDH2 mRNA were examined by qRT-PCR. Low level = mRNA expression in EC over benign samples (fold over $\mathrm{NE}) \leq 0,5$.

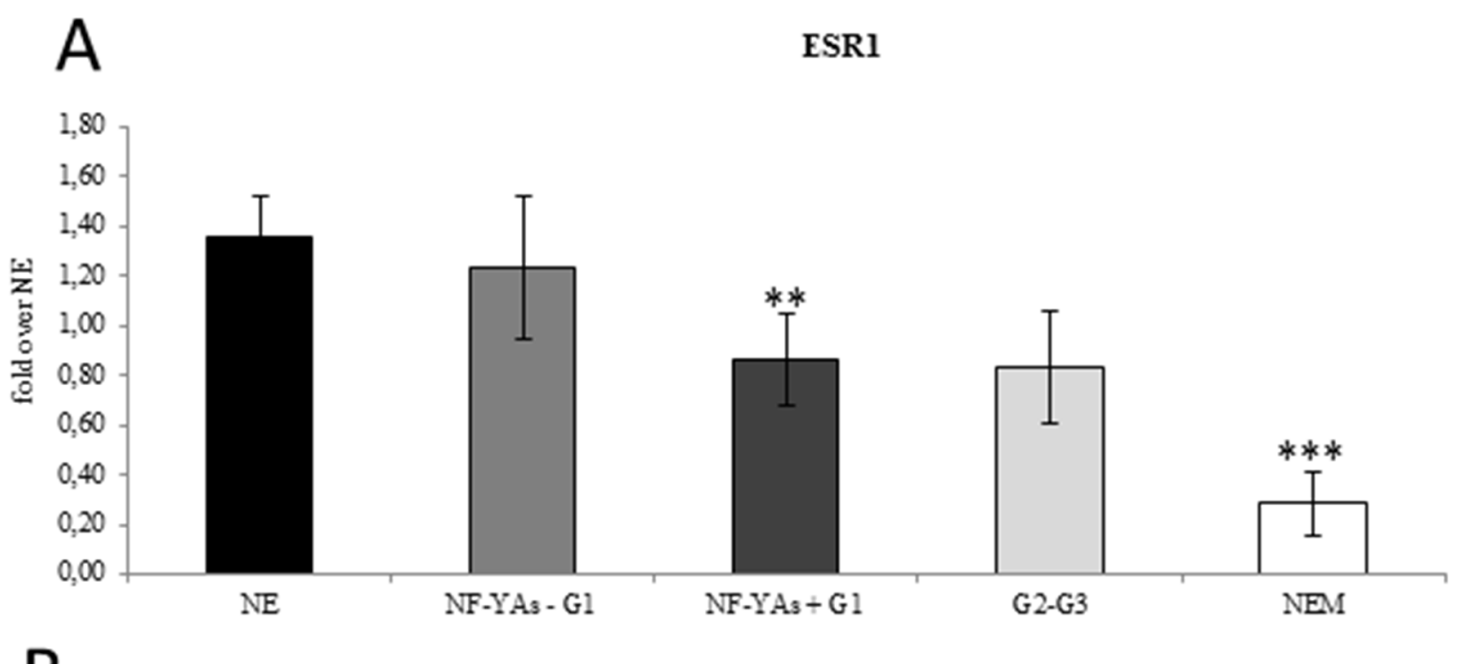

B

\section{$\mathrm{CDH} 2$}

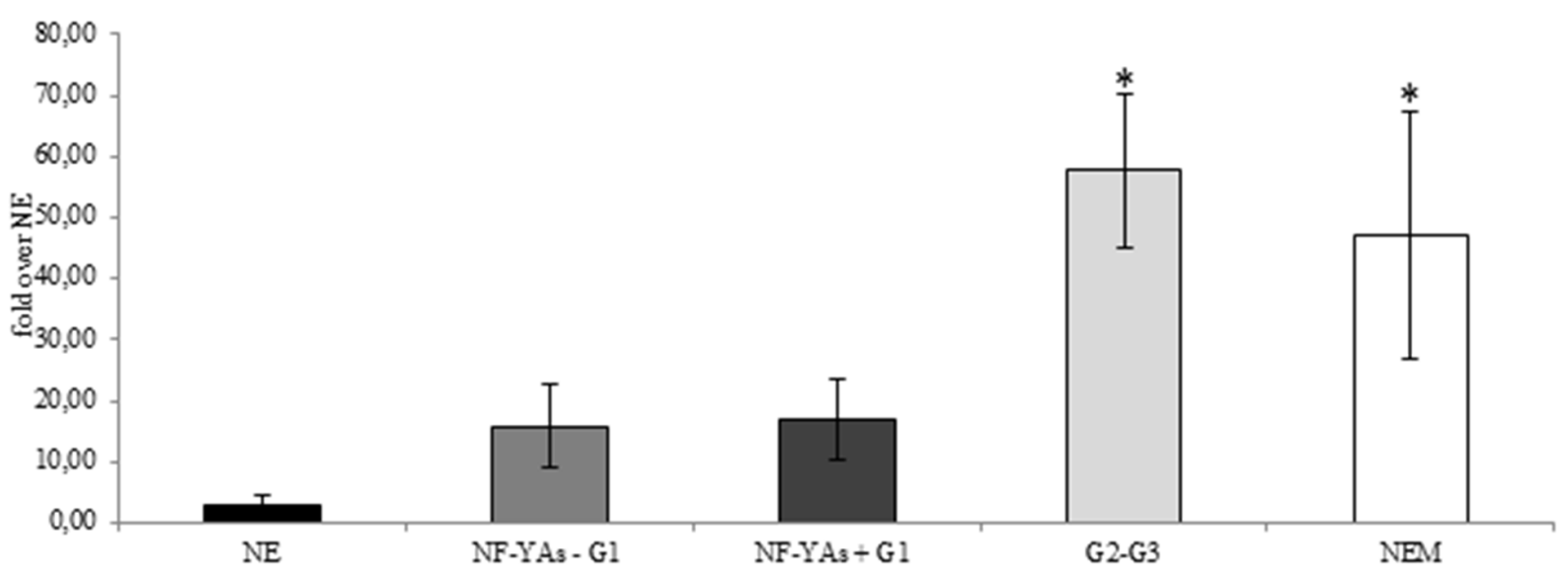

Figure 2: ESR1 decreased mRNA levels are associated with increase CDH2 expression. Average of expression of the ESR-1 mRNA A., and of CDH2 B. mRNA in EC tissues examined by qRT-PCR \pm SD. mRNA expression was normalized for $18 \mathrm{~S}$ rRNA levels as endogenous control. Statistical significance: $* \mathrm{P} \leq 0.05, * * \mathrm{P} \leq 0.01, * * * \mathrm{P} \leq 0.001$ vs NE. The error bars indicate the standard error. 
tissues, while NF-YA long isoform (NF-YAl) is always expressed, whereas NF-YAs was consistently expressed in high grade G2/G3 EEC and in NEM subtypes. Interestingly, in low grade G1 EECs a heterogeneous expression of NF-YAs was observed with some samples expressing exclusively the NF-YAl and others samples expressing both isoforms. Based on this observation we are now able to stratify low grade G1 EEC in two subgroups: one expressing only NF-YAl, NF-YAs negative (NF-YAs-), and another expressing both
A

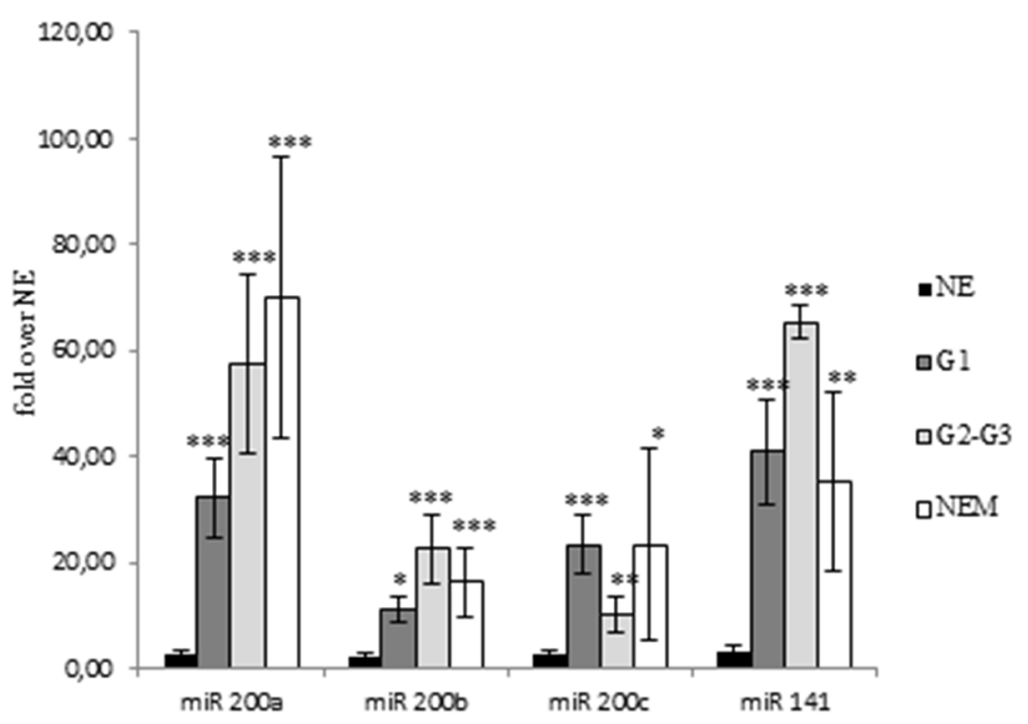

$\mathrm{C}$

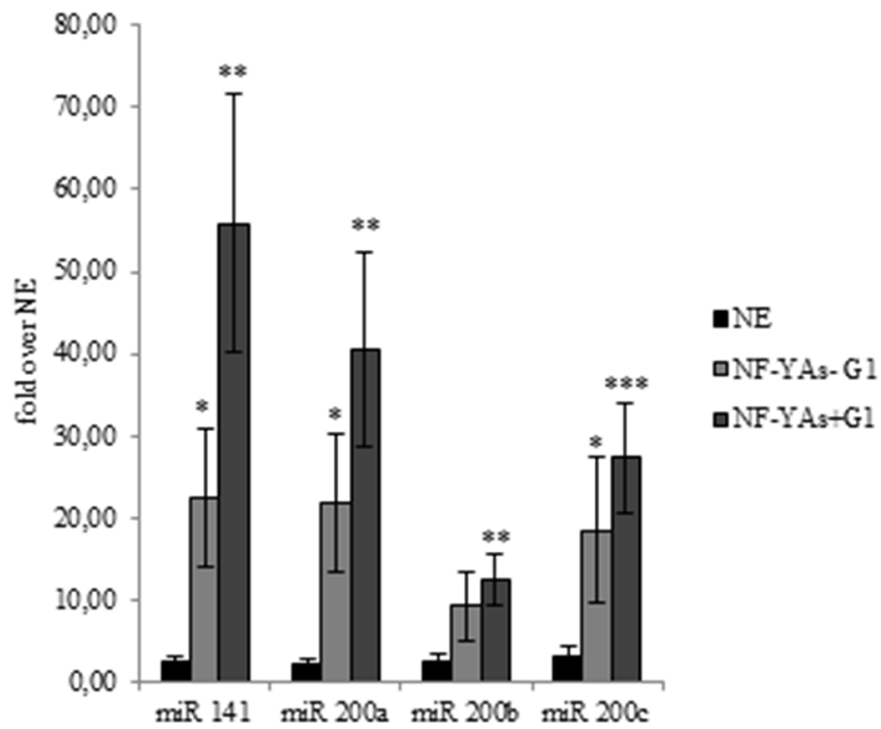

B
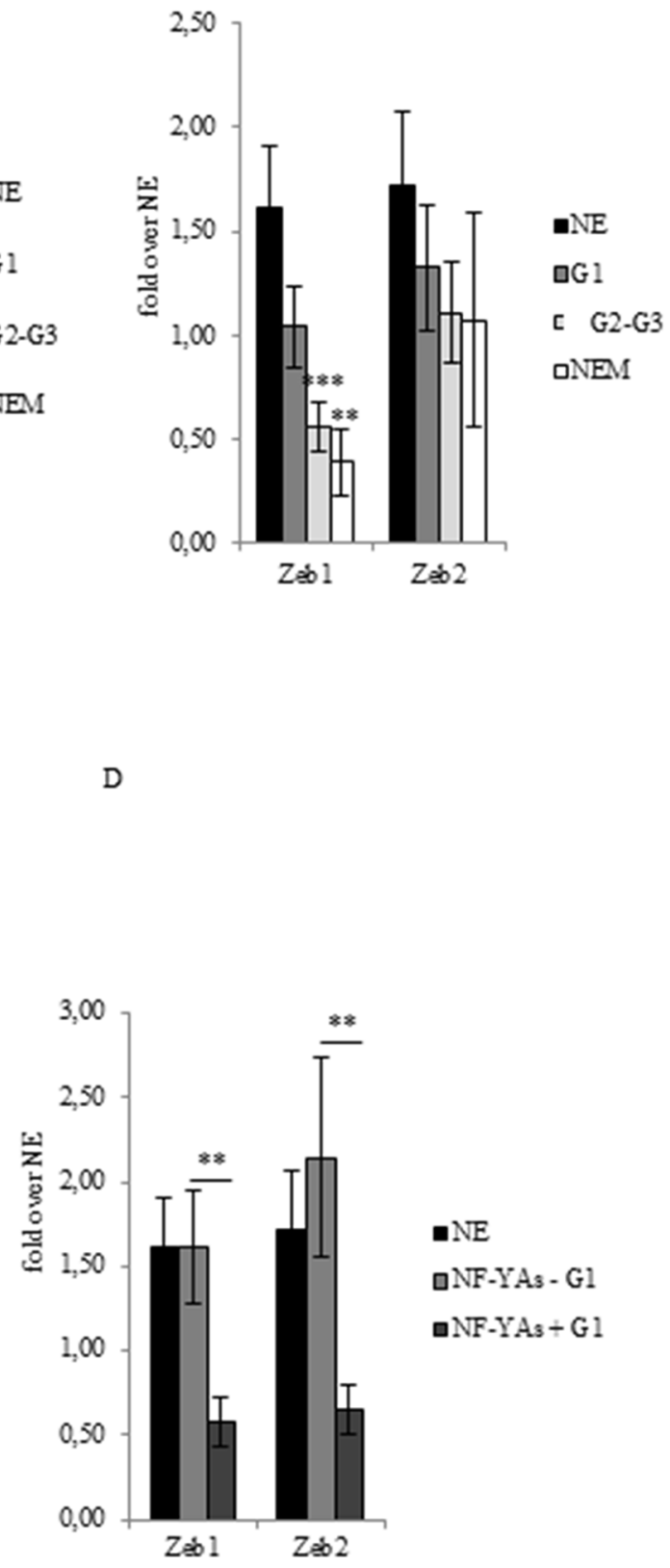

Figure 3: miR-200s and ZEBs expression in EC FFPE tissues. Average of miR-200 family members (miR-200a, miR-200b, miR200c, and miR-141) expression A, of ZEB1 and ZEB2 B, mRNA in benign (NE), G1 EEC, G2-G3 EEC, and NEM FFPE tissues. Average of miR-200 family members (miR-200a, miR-200b, miR-200c, and miR-141) expression C, and of ZEB1 and ZEB2 D, mRNA in benign (NE), NF-YAs positive (NF-YA+) and negative (NF-YAs-) G1 subsets of EEC FFPE tissues. Analysis was performed by qRT-PCR \pm SD. miRNA expression was normalized using small nuclear RNA U6 as endogenous control. mRNA expression was normalized for 18S rRNA levels as endogenous control. Statistical significance: ${ }^{*} \mathrm{P} \leq 0.05, * * \mathrm{P} \leq 0.01, * * * \mathrm{P} \leq 0.001$, The error bars indicate the standard error. 
isoforms, NF-YAs positive (NF-YAs + ). The exclusive presence of NF-YAl isoform in benign tissues suggests that it may represent a marker of differentiation and that the presence of NF-YAs may be linked with an increase of a pool of poorly differentiated cells in tumors tissues.

To better characterize the significance of the heterogeneous expression of NF-YA isoforms in G1 EEC, we analyzed the estrogen receptor (ER) status. It has been well documented that higher level of ERs are significantly associated with good prognosis, and that early stage-well differentiated EC usually retain their expression, whereas poorly differentiated tumors often lack one or both of these receptors [18] and [26]. In the current study we first confirmed this correlation, than by clustering of samples expressing and not expressing NF-YAs in G1 EEC we highlight a strong correlation between loss of ERs and presence of NF-YAs.

\section{A}

LMNA

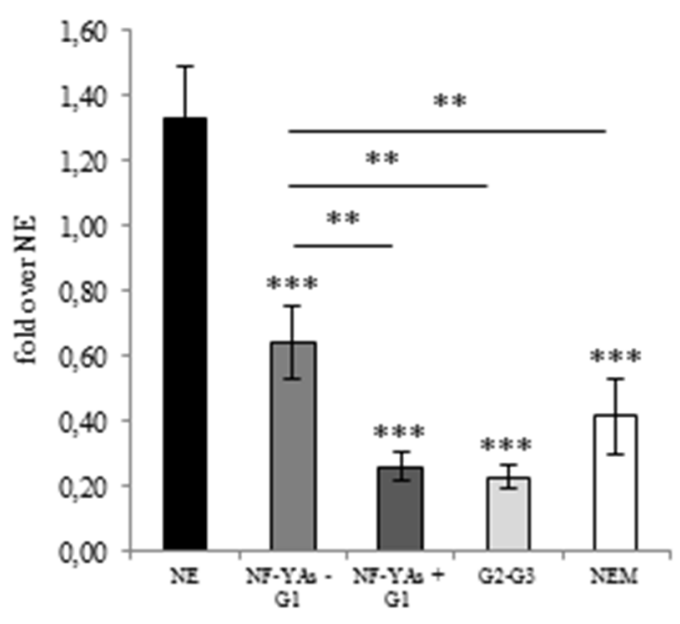

B

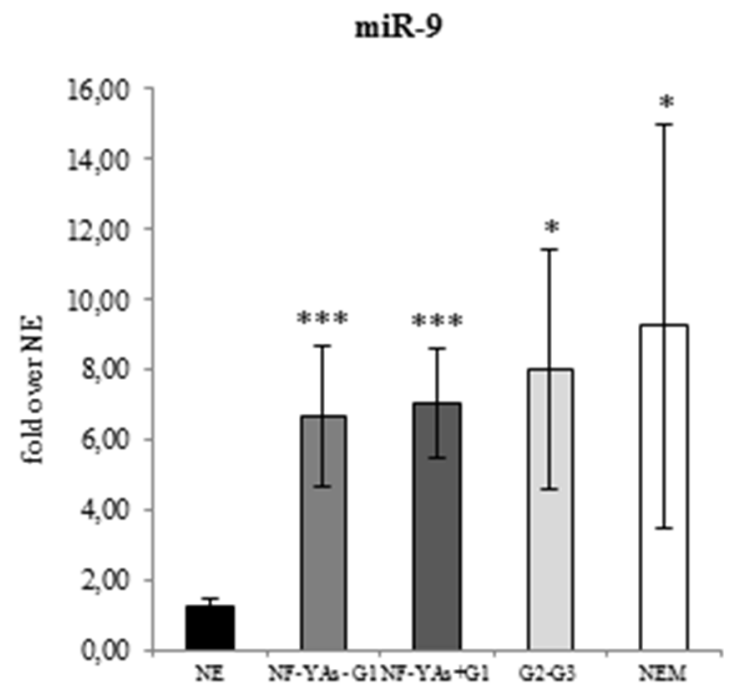

Figure 4: Evaluation of lamin A and mir-9 expression in ECs. Average of expression of LMNA mRNA A. and miR-9 B. in benign (NE), in the two subsets of FFPE G1 EEC tissues (NF-YAs- and NF-YAs+) G1 EEC, G2-G3 EEC, and NEM FFPE tissues examined by qRT-PCR \pm SD. mRNA expression was normalized for $18 \mathrm{~S}$ rRNA levels. miRNA expression was normalized using small nuclear RNA U6 as endogenous control. Statistical significance: ${ }^{*} \mathrm{P} \leq 0.05, * * \mathrm{P} \leq 0.01, * * * \mathrm{P} \leq 0.001$. The error bars indicate the standard error. 


\begin{tabular}{|c|c|}
\hline NF-YAs fw & ACAGATTCAGCAGCAGGTCC \\
\hline NF-YAs rv & ATGGGTTGGCCAGTTGATGT \\
\hline NF-YAl fw & CAGGGTGGTGTCACTGCTG \\
\hline NF-YAl rv & TACCTGGAGGGTCTGGACTT \\
\hline LMNA fw & GGACAATCTGGTCACCCGC \\
\hline LMNA rv & TGGCAGGTCCCAGATTACATG \\
\hline ESR1 fw & TACTGACCAACCTGGCAGACAG \\
\hline ESR1 rv & TGGACCTGATCATGGAGGGT \\
\hline ESR2 fw & AGTTGGCCGACAAGGAGTTG \\
\hline ESR2 rv & CGCACTTGGTCGAACAGG \\
\hline ZEB1 fw & AACCACCCTTGAAAGTGATCCA \\
\hline ZEB1 rv & CTTGTCTTTCATCCTGATTTCCATT \\
\hline ZEB2 fw & CAAAGGAGAAAGTACCAGCGGA \\
\hline ZEB2 rv & CATCAAGCAATTCTCCTGAAATCC \\
\hline CDH1 fw & CCCACCACGTACAAGGGTC \\
\hline CDH1 rv & ATGCCATCGTTGTTCACTGGA \\
\hline $\mathrm{CDH} 2 \mathrm{fw}$ & AGAAGAAGACCAGGACTATGACTTGAG \\
\hline $\mathrm{CDH} 2 \mathrm{rv}$ & ACAGTGTCAGGCTGCTGCAG \\
\hline 18S rRNA fw & CCTGGATACCGCAGCTAGGA \\
\hline 18S rRNA rv & GCGGCGCAATACGAATGCCCC \\
\hline
\end{tabular}

A recent study has reported that lack of ER- $\alpha$ correlates with epithelial mesenchymal transition and metastasis [26]. In agreement, ERs regulate transcription of miR-200 family members, a family of miRNAs that are known to maintain an epithelial phenotype in several cancers among which in low grade EEC [31], [32], [33], [34] and [35]. In G1 EEC we observed an upregulation of miR-200 family members. A significant increase of $\mathrm{N}$-cadherin mRNA and a lower E-cadherin/N-cadherin index was observed in more aggressive EC tissues, thus confirming the association of high EMT signature with tumor aggressiveness and poor prognosis. However, our analysis does not revealed a correlation between $\mathrm{N}-\mathrm{Cadherin,} \mathrm{E-Cadherin} \mathrm{and} \mathrm{NF-YAs} \mathrm{expression} \mathrm{in} \mathrm{low}$ grade G1 EEC.

A retrospective analysis in our cohort of low grade G1 EEC revealed that the two patients developing liver and lung recurrences both belonged to the NF- YAs positive subgroup. Considering the relative rarity of distant metastasis in G1 EEC $(<5 \%)$, and our low number of cases, further studies are needed to evaluate the possible correlation between NF-YAs expression and risk of recurrence.

Lamin A expression frequently correlates with cancer subtypes and cancer aggressiveness, proliferative capacity and differentiation state [22], [23], [24] and [25]. Recently, we reported that alteration of lamin A levels may be use as prognostic biomarker in EC and a correlation of its decreased expression levels with an increased myometrial infiltration in early stages of EEC [25]. Very interestingly, we observed an inverse correlation between NF-YAs and lamin A expression, thus further supporting the hypothesis of a possible involvement of NF-YAs in tumor differentiation and aggressiveness. In agreement with this, we have recently characterized a role for lamin A in counteracting NF-Y transcriptional activity in cancer cells [36]. Thus, we hypothesizes that concomitant expression of NF-YAs and lamin A down-modulation may be associated with an increased proliferation rate and a more invasive phenotype of cancer cells.

Further investigations are needed to confirm and better characterize our findings, considering also the new genomic characterisation of EC recently presented by the Cancer Genome Atlas Research Network (TCGA) [37].

In conclusion, our data highlight that a combination of NF-YAs, lamin A and ERs expression could be a novel potential predictive signature for new stratification approaches in low grade G1 ECs. 


\section{MATERIAL AND METHODS}

\section{Patient cohort}

A retrospective cohort of formalin-fixed, paraffinembedded (FFPE) specimens from patients with EC $(n=87)$ and NE specimens (FFPE) from patients who underwent a hysterectomy to treat other benign disease $(n=13)$ were collected. According with the histologic grade, we analysed 29 low grade (G1), 49 high grade (G2/ G3) ECs and 9 non endometrioid tissues (NEM). 79,6\% of high grade $(\mathrm{G} 2 / \mathrm{G} 3)$ patients developed metastasis (Table 1). Biopsies were sampled for primary tumors in hysterectomy specimens.

\section{RNA extraction and RT-PCR}

Total RNA derived from FFPE tissues was extracted using the PureLink ${ }^{\mathrm{TM}}$ FFPE Total RNA Isolation Kit (Invitrogen) following the manufacturer's instructions and reverse-transcribed using PrimeScript RT reagent kit (Takara). The quality of the total RNA was measured using a NanoDrop 2000 spectrophotometer (Thermo Fisher Scientific, Wilmington DE, USA). Quantitative PCR (qPCR) was performed using SYBR Select (Applied Biosystems) on an ABI Prism 7500 apparatus (Applied Biosystems). mRNA expression was normalized for $18 \mathrm{~S}$ rRNA levels. Relative mRNA expression was calculated using the comparative $\mathrm{Ct}$ method $(2-\Delta \Delta \mathrm{Ct})$. Primers used are listed in Table 3.

\section{MicroRNA analysis}

Reverse transcription and qRT-PCR amplification were performed in two steps. In the first reverse transcription step, $10 \mathrm{ng}$ of RNA was used in reactions with specific stem-loop RT primer for miR-200a, miR200b, miR-200c, miR-141, and miR-9 and endogenous control primer for small nuclear RNA U6. Reaction was performed with TaqMan MicroRNA Reverse Transcription Kit, according to the manufacturer's protocol (Applied Biosystems, Foster City, CA). In the second step, cDNA samples were amplified in Real Time PCR instrument 7500 (Applied Biosystems) with the specific TaqMan miR-200a, miR-141, and miR205 assay and small nuclear RNA U6 as endogenous control. The relative quantity (RQ) of each miRNA was calculated by the comparative CT (2- $\Delta \Delta \mathrm{CT})$ method, in which $\Delta \Delta \mathrm{CT}$ was calculated as follows: $\Delta \Delta \mathrm{CT}=$ (CTmiR-of-interest - CTU6)cancer - (CTmiR-ofinterest - CTU6)benign.

\section{Immunoblotting}

The paraffin from thin sections of FFPE specimens was melted at $72^{\circ} \mathrm{C}$ for 20 minutes using heat in the presence of a specially designed Melting Buffer contained in the PureLink ${ }^{\mathrm{TM}}$ FFPE Isolation Kit used for RNA extraction (Invitrogen). Tissues were then separated from the melted paraffin by centrifugation. Proteins were extracted in a high $\mathrm{pH}$ lysis buffer (20 $\mathrm{mM}$ Tris $\mathrm{HCl} \mathrm{pH}$ 9.0, 0.2 M Glycine, $2 \%$ (w/v) SDS). The samples were first incubated on ice for $5 \mathrm{~min}$, and mixed by vortexing, then boiled at $100^{\circ} \mathrm{C}$ for $20 \mathrm{~min}$ followed by an 1 hour incubation at $80^{\circ} \mathrm{C}$ for 2 hours. After extraction, any remaining unsolubilized material was pelleted at $14000 \times \mathrm{g}$ for 20 minutes, and protein concentration of total protein extracted was determined by the BCA Protein Assay (Pierce Chemicals Co., Rockford, IL, USA). The Pierce BCA Protein Assay is a detergent compatible formulation and the protein standards were prepared using the same lysis buffer as the samples. Proteins were resolved by SDS-PAGE and electrotransferred to nitrocellulose. Each membrane was blocked with $5 \%$ non-fat dry milk in Tris buffered saline-Tween-20 (TBST) for 1 hour at room temperature and subsequently incubated with primary antibody for 16 hours at $4{ }^{\circ} \mathrm{C}$. The following antibodies were used: anti-NF-YB monoclonal (Santa Cruz), anti-NF-YA monoclonal (Santa Cruz), anti-Lamin A (Santa Cruz), and anti- $\beta$ actin (sigma-aldrich). Immunoreactivity was detected by sequential incubation with HRP-conjugated secondary antibody.

\section{Statistical analysis}

Data were reported as mean and standard deviation. In all experiments, comparisons of results from qRT-PCR between two groups were based on Student's t-test and one-way analysis of variance (ANOVA). $\mathrm{P} \leq 0.05$ was deemed to be significant variation.

\section{Abbreviations}

Endometrial cancer $=\mathrm{EC}$

Endometrioid endometrial adenocarcinoma $=\mathrm{EEC}$ Non endometrioid endometrial adenocarcinoma $=$ NEM

Benign tissue $=\mathrm{NE}$

Formalin-fixed, paraffin-embedded $=$ FFPE

NF-YA short form $=$ NF-YAs

NF-YA long form $=$ NF-YAl

Estrogen receptor $=\mathrm{ER}$

Epithelial-mesenchymal transition $=$ EMT

\section{ACKNOWLEDGMENTS AND GRANT SUPPORT}

This work has been founded by Italian Association for Cancer Research (AIRC) (IG n. 13234 to G.P.) and IRE Internal projects to E.V. 


\section{CONFLICT OF INTERESTS}

The authors report no conflicts of interest. The authors are responsible for the content and writing of the paper.

\section{REFERENCES}

1. Amant F, Moerman P, Neven P, Timmerman D, Van Limbergen E, Vergote I. Endometrial cancer. Lancet 2005; 388:491-505.

2. Rose PG: Endometrial carcinoma. N Engl J Med. 1996; 9:640-49.

3. Creasman WT. Prognostic significance of hormone receptors in endometrial cancer. Cancer 1993; 4:1467-70.

4. Morrow CP, Bundy BN, Kurman RJ, Creasman WT, Heller P, Homesley HD, Graham JE. Relationship between surgicalpathological risk factors and outcome in clinical stage I and II carcinoma of the endometrium: a Gynecologic Oncology Group study. Gynecol Oncol 1991; 55-65.

5. Prat J. Prognostic parameters of endometrial carcinoma. Hum Pathol. 2004;6:649-62.

6. Salvesen HB, Haldorsen IS, Trovik J. Markers for individualised therapy in endometrial carcinoma. Lancet Oncol. 2012; 13:e353-61.

7. Dolfini D, Mantovani R. Targeting the Y/CCAAT box in cancer: YB-1 (YBX1) or NF-Y? Cell Death Differ. 2013; 20:676-85.

8. Di Agostino S, Strano S, Emiliozzi V, Zerbini V, Mottolese M, Sacchi A, Blandino G, Piaggio G. Gain of function of mutant $\mathrm{p} 53$ - the mutant $\mathrm{p} 53 / \mathrm{NF}-\mathrm{Y}$ protein complex reveals an aberrant transcriptional mechanism of cell cycle regulation. Cancer Cell. 2006; 10:191-202.

9. Gurtner A, Fuschi P, Magi F, Colussi C, Gaetano C, Dobbelstein M, Sacchi A, Piaggio G. NF-Y dependent epigenetic modifications discriminate between proliferating and postmitotic tissue. PLoS One. 2008; 3:e2047.

10. Manni I, Caretti G, Artuso S, Gurtner A, Emiliozzi V, Sacchi A, Mantovani R, Piaggio G. Posttranslational regulation of NF-YA modulates NF-Y transcriptional activity. Mol Biol Cell. 2008; 19:5203-13.

11. Ishimaru F, Mari B, Shipp MA. The type 2 CD10/neutral endopeptidase 24.11 promoter: functional characterization and tissue-specific regulation by $\mathrm{CBF} / \mathrm{NF}-\mathrm{Y}$ isoforms. Blood. 1997; 89:4136-45.

12. Ceribelli M, Benatti $\mathrm{P}$, Imbriano $\mathrm{C}$, Mantovani R. NF-YC complexity is generated by dual promoters and alternative splicing. J Biol Chem. 2009; 284:34189-20014.

13. Grskovic M, Chaivorapol C, Gaspar-Maia A, RamalhoSantos M. Systematic identification of cis-regulatory sequences active in mouse and human embryonic stem cells. PLos Genet. 2007; 3:e145.

14. Pappa KI, Polyzos A, Jacob-Hirsch J, Amariglio N, Vlachos GD, Loutradis D, Anagnou NP. Profiling of Discrete Gynecological Cancers Reveals Novel Transcriptional
Modules and Common Features Shared by Other Cancer Types and Embryonic Stem Cells. PLoS One. 2015; 10:e0142229.

15. Zhang Y, Zhao D, Gong C, Zhang F, He J, Zhang W, Zhao Y, Sun J. Prognostic role of hormone receptors in endometrial cancer: a systematic review and meta-analysis. World J Surg Oncol. 2015; 13:208.

16. Backes FJ, Walker CJ, Goodfellow PJ, Hade EM, Agarwal G, Mutch D, Cohn DE, Suarez AA. Estrogen receptor-alpha as a predictive biomarker in endometrioid endometrial cancer. Gynecol Oncol. 2016; 141:312-7.

17. Utsunomiya H, Suzuki T, Harada N, Ito K, Matsuzaki S, Konno R, Sato S, Yajima A, Sasano H. Analysis of estrogen receptor alpha and beta in endometrial carcinomas: correlation with ER beta and clinicopathologic findings in 45 cases. IntJ Gynecol Pathol. 2000; 4:335-41.

18. Weihua Z, Saji S, Makinen S, Cheng G, Jensen EV, Warner M, Estrogen receptor (ER) beta, a modulator of ERalpha in the uterus. Proc Natl Acad Sci USA. 2000; 11:5936-41.

19. Thomas C, Gustafsson JA. The different roles of ER subtypes in cancer biology and therapy. Nat Rev Cancer 2011; 597-608.

20. Dong Y, Si JW, Li WT, Liang L, Zhao J, Zhou M, et al. miR200a/miR-141 and miR-205 upregulation might be associated with hormone receptor status and prognosis in endometrial carcinomas. Int J Clin Exp Pathol. 2015; 8:2864-75.

21. Foster CR, Przyborski SA, Wilson RG, Hutchison CJ. Lamins as cancer biomarkers J Biochem Soc Trans. 2010; 38:297-300.

22. Prokocimer M, Davidovich M, Nissim-Rafinia M, WieselMotiuk N, Bar DZ, Barkan R, Meshorer E, Gruenbaum Y. Nuclear lamins- key regulators of nuclear structure and activities. J Cell Mol Med. 2009; 13:1059-85.

23. Capo-chichi CD, Cai KQ, Simpkins F, Ganjei-Azar P, Godwin AK, Xu XX. Nuclear envelope structural defects cause chromosomal numerical instability and aneuploidy in ovarian cancer. BMC Med. 2011; 9-28.

24. Capo-chichi CD, Cai KQ, Smedberg J, Ganjei-Azar P, Godwin AK, Xu XX. Loss of A-type lamin expression compromises nuclear envelope integrity in breast cancer. Chin J Cancer. 2011; 30:415-25.

25. Cicchillitti L, Corrado G, Carosi M, Loria R, Dabrowska ME, Trojano G, Mancini E, Cutillo G, Falcioni R, Piaggio G, Vizza E. Altered lamin A expression as a possible prognostic biomarker in endometrioid endometrial cancers. Italian J of Gyn \& Obst,. 2016; 28:57-65.

26. Wik E, Ræder MB, Krakstad C, Trovik J, Birkeland E, Hoivik EA, Mjos S, Werner HM, Mannelqvist M, Stefansson IM, Oyan AM, Kalland KH, Akslen LA, et al. Lack of estrogen receptor- $\alpha$ is associated with epithelialmesenchymal transition and PI3K alterations in endometrial carcinoma. Clin Cancer Res. 2013; 19:1094-105.

27. Panda H, Pelakh L, Chuang TD, Luo X, Bukulmez O, Chegini N. Endometrial miR-200c is altered during 
transformation into cancerous states and targets the expression of ZEBs, VEGFA, FLT1, IKK $\beta$, KLF9, and FBLN5. Reprod Sci. 2012; 19:786-96.

28. Krasner C. Aromatase inhibitors in gynecologic cancers. J Steroid Biochem Mol Biol. 2007; 106:76-80.

29. Dong Y, Si JW, Li WT, Liang L, Zhao J, Zhou M, Li D, Li T. miR-200a/miR-141 and miR-205 upregulation might be associated with hormone receptor status and prognosis in endometrial carcinomas. Int J Clin Exp Pathol. 2015; 8:2864-75.

30. Myatt SS, Wang J, Monteiro LJ, Christian M, Ho KK, Fusi L, Dina RE, Brosens JJ, Ghaem-Maghami S, Lam EW. Definition of microRNAs that repress expression of the tumor suppressor gene FOXO1 in endometrial cancer. Cancer Res. 2010; 70:367-77.

31. Snowdon J, Zhang X, Childs T, Tron VA, Feilotter H. The microRNA-200 family is upregulated in endometrial carcinoma. PLoS One. 2011; 6:e22828.

32. Lee JW, Park YA, Choi JJ, Lee YY, Kim CJ, Choi C, Kim TJ, Lee NW, Kim BG, Bae DS. The expression of the miRNA-200 family in endometrial endometrioid carcinoma. Gynecol Oncol. 2011; 120:56-62.

33. Mueller DW, Rehli M, Bosserhoff AK. miRNA expression profiling in melanocytes and melanoma cell lines reveals miRNAs associated with formation and progression of malignant melanoma. J Invest Dermatol. 2009; 129:1740-51.
34. Elson-Schwab I, Lorentzen A, Marshall CJ. MicroRNA-200 family members differentially regulate morphological plasticity and mode of melanoma cell invasion. PLoS One 2010; 5:e13176.

35. Pan Y, Liang H, Chen W, Zhang H, Wang N, Wang F, Zhang S, Liu Y, Zhao C, Yan X, Zhang J, Zhang CY, Gu H, et al. microRNA-200b and microRNA-200c promote colorectal cancer cell proliferation via targeting the reversion-inducing cysteine-rich protein with Kazal motifs. RNA Biol. 2015; 12:276-89.

36. Cicchillitti L, Manni I, Mancone C, Regazzo G, Spagnuolo M, Alonzi T, Carlomosti F, Dell'Anna ML, Dell'Omo G, Picardo M, Ciana P, Capogrossi MC, Tripodi M, et al. The laminA/NF-Y protein complex reveals an unknown transcriptional mechanism on cell proliferation. Oncotarget. 2016; 8:2628-2646. doi: 10.18632/oncotarget.12914.

37. Cancer Genome Atlas Research Network, Kandoth C, Schultz N, Cherniack AD, Akbani R, Liu Y, Shen H, Robertson AG, Pashtan I, Shen R, Benz CC, Yau C, Laird PW, Ding L, Zhang W, Mills GB, Kucherlapati R, Mardis $\mathrm{ER}$, Levine DA. Integrated genomic characterization of endometrial carcinoma. Nature 2013; 497:67-73. Erratum in: Nature. 2013; 500:242. 